\title{
Effects of strong anchoring on the dynamic moduli of heterogeneous nematic polymers II: Oblique anchoring angles
}

\author{
Eric P. Choate \\ Department of Mathematics, \\ University of North Carolina at Chapel Hill \\ Chapel Hill, NC 27599-3250 \\ M. Gregory Forest \\ Department of Mathematics, \\ Institute for Advanced Materials \\ University of North Carolina at Chapel Hill \\ Chapel Hill, NC 27599-3250 \\ Lili Ju \\ Department of Mathematics, \\ Interdisciplinary Mathematics Institute, \\ University of South Carolina \\ Columbia, SC 29208
}

August 28, 2009 


\begin{abstract}
We extend previous work on the linear viscoelastic moduli of heterogeneous nematic polymers in a small amplitude oscillatory shear flow, focusing on the role of the orientational anchoring conditions at the plates. When tangential or normal anchoring conditions are applied, the Doi-Marrucci-Greco orientation tensor-flow model effectively reduces to the Leslie-Ericksen director-flow model, predicting that director distortions control the dynamic moduli with negligible contributions from tensor order parameters. In this paper, we examine oblique anchoring angles. We use a combination of analysis and numerical simulation on the generalized tensor-flow system for arbitrary anchoring conditions to show that any oblique anchoring condition induces a nontrivial order parameter contribution to the dynamic moduli, which vanishes only in the limit of tangential or normal anchoring. Our approach reveals that the storage and loss moduli admit an approximate decomposition in terms of two reduced problems that are exactly solvable: the heterogeneous director-flow response plus the monodomain tensor response to an imposed shear. The importance of this result is that we gain scaling properties of the moduli with respect to material parameters and experimental conditions without having to compute and assimilate across the full parameter space. These results provide insight into the relative importance of the distortional versus bulk nematic elastic stress in determining the viscoelastic moduli, predicting that anchoring conditions tune the relative contributions.
\end{abstract}




\section{Introduction}

Orientational equilibria of a nematic liquid crystal polymer system select the degree of order (the so-called order parameters) but the principal direction of order is degenerate in the absence of an external field. The addition of flow and heterogeneity to the model breaks this orientational degeneracy. In previous experimental results [Mendil et al., 2005, 2006; PujolleRobic and Noirez, 2001] and modeling studies [Choate et al., 2008], the legacy of the director degeneracy in the equilibrium conditions at solid walls is that rheological properties can vary significantly. Said differently, the chemical or mechanical treatment of solid boundaries can affect the linear viscoelastic response of the entire sample, far from a local boundary layer. As in [Choate et al., 2008], we model the orientational degeneracy through the anchoring conditions on two parallel plates in a shear cell, and then observe its effect on the storage and loss moduli by solving the model equations for small amplitude oscillatory shear flow with one-dimensional heterogeneity.

In our earlier paper, we showed that tangential and normal anchoring of the director with respect to the plates leads to significant simplifications of the governing flow-orientation equations, which we then solved to elicit linear viscoelastic moduli for both conditions. The point of this paper is to solve the more general equations and interpret the results for anchoring conditions that interpolate between these two limits.

We use the second moment tensor $\mathbf{M}$ of the orientational probability density function $f$, or its traceless part $\mathbf{Q}=\mathbf{M}-\frac{\mathbf{I}}{3}$, as our primitive variable for the orientational distribution of nematic liquid crystal polymers. The degeneracy is highlighted by the spectral representation $\mathbf{Q}=s\left(\mathbf{n}_{1} \mathbf{n}_{1}-\frac{\mathbf{I}}{3}\right)+\beta\left(\mathbf{n}_{2} \mathbf{n}_{2}-\frac{\mathbf{I}}{3}\right)$, where $s$ and $\beta$ are order parameters measuring the birefringence relative to the eigenvector frame $\mathbf{n}_{1}$ (the major director), $\mathbf{n}_{2}$, and $\mathbf{n}_{3}$. When there is no flow and the dimensionless strength of the excluded volume potential $N$ is sufficiently high $(N>3)$, there is an uniaxial equilibrium orientation $\mathbf{Q}_{0}=s_{0}\left(\mathbf{n}_{0} \mathbf{n}_{0}-\frac{\mathbf{I}}{3}\right)$ with

$\beta=0$ because of uniaxiality. The equilibrium order parameter $s_{0}=\frac{1}{4}\left(1+3 \sqrt{1-\frac{8}{3 N}}\right)$ determines the degree of ordering; however, the degeneracy is manifested in that the major director $\mathbf{n}_{0}$ is arbitrary.

If we impose strong nematic anchoring conditions $\mathbf{Q}\left(y= \pm \frac{1}{2}\right)=\mathbf{Q}_{0}$, we may choose any major director anchoring condition $\mathbf{n}_{0}$, and we parametrize this degeneracy with the director anchoring angle $\psi_{0}$ so that $\mathbf{n}_{0}=\left(\cos \psi_{0}, \sin \psi_{0}, 0\right)$. When there is no flow, this degeneracy is extended throughout the system with the homogeneous solution $\mathbf{Q}(y) \equiv \mathbf{Q}_{0}$. This paper examines the effect of this angle when we impose small amplitude oscillatory shear driving conditions.

In [Choate et al., 2008], we found that for parallel or normal anchoring conditions, the leading order system for the velocity, director angle, and order parameters diagonalizes: The primary velocity and director angle decouple from the order parameters, and we recover the Leslie-Ericksen (LE) model for small amplitude oscillatory shear which is explicitly solvable [Burghardt, 1991; de Andrade Lima and Rey, 2006]. The order parameter contribution is zero at leading order. The upshot is that for these special anchoring angles, the distortional elastic stress is dominant over the nematic (bulk monodomain) elastic stress, which has consequences for the frequency-dependent scaling behavior of the storage and loss moduli. In essence, nematic polymers behave like small molecule liquid crystals for these two anchoring 
conditions in the presence of one-dimensional heterogeneity between the parallel plates.

In a complimentary study [Choate and Forest, 2006], we examined the monodomain limit in which the orientation was assumed to be homogeneous and the velocity profile linear, and as a consequence, the distortional elastic stress is assumed to be zero. At leading order, the order parameter system was analytically solvable for an arbitrary director angle $\psi_{0}$, and the nematic elastic stress was found to be proportional to $\sin ^{2} 2 \psi_{0}$. Thus, the nematic elastic stress was zero for normal and tangential anchoring just as it was in the heterogeneous case, but it is nonzero for oblique anchoring. This again has implications for dynamic moduli.

The present paper examines the combination of these two effects to gain further insight into the role that the director anchoring angle plays in determining the storage and loss moduli. In any physical system where there are curved boundaries, there will be an effective oblique anchoring condition, which is the motivation for the present study. We generalize [Choate et al., 2008] by computing the stress-strain relationship from the full orientation tensor and flow model with oblique anchoring angles, and then we compare the moduli predictions with the limiting cases of tangential and normal anchoring. Remarkably, we find that the linear viscoelastic response is well approximated by a superposition of a directordominated heterogeneous contribution (which is the Leslie-Ericksen model with oblique anchoring) and a tensor-dominated homogeneous contribution (which we solved previously for oblique anchoring).

\section{Model formulation and dimensional analysis}

We use the same nondimensionalization as [Choate et al., 2008] to examine oscillatory shear flow between two parallel plates separated by the distance $h$, which forms the characteristic length for our one spatial dimension $y$. To better compare with results from Leslie-Ericksen theory [Burghardt, 1991; de Andrade Lima and Rey, 2006], we choose the characteristic Frank stress $\tau_{F}=\frac{K}{h^{2}}=\frac{\nu k T N \mathcal{L}^{2} s_{0}^{2}}{8 h^{2}}$ and Leslie viscosity $\eta_{0}=\frac{\nu k T s_{0}^{2}}{D_{r}}$, where $K$ is the Frank elasticity constant, $\nu$ is the polymer number density, $k$ is the Boltzmann constant, $T$ is the temperature, $\mathcal{L}$ is the penetration depth of the elasticity potential, and $D_{r}$ is the rotational diffusion rate. Their ratio defines the characteristic timescale $t_{0}=\frac{\eta_{0}}{\tau_{F}}=\frac{8 h^{2}}{N \mathcal{L}^{2} D_{r}}$. The velocity scale is $\frac{h}{t_{0}}$, and the dimensionless rate-of-strain and vorticity tensors are $\mathbf{D}=\frac{1}{2}\left(\nabla \mathbf{v}+\nabla \mathbf{v}^{T}\right)$ and $\boldsymbol{\Omega}=\frac{1}{2}\left(\nabla \mathbf{v}-\nabla \mathbf{v}^{T}\right)$, respectively.

An oscillatory shear flow may be driven by imposing either the velocity at the boundary $v_{x}\left(y= \pm \frac{1}{2}\right)= \pm v_{0} \cos \omega t$ or the shear stress $\tau_{x y}=\tau_{0} \cos \omega t$. These driving conditions lead to two different but related nondimensional parameters. The Ericksen number Er is the ratio of the flow-induced viscous stress to the Frank stress, and the Deborah number De is the ratio of the characteristic shear rate to the rotational diffusion rate. If the shear stress is imposed, $E r=\frac{\tau_{0}}{\tau_{F}}$ and $D e=\frac{\dot{\gamma}_{e f f}}{D_{r}}$, where $\dot{\gamma}_{e f f}=\frac{\tau_{0}}{\eta_{0}}$ defines an effective shear rate. For imposed velocity boundary conditions, $E r=\frac{\tau_{e f f}}{\tau_{F}}$ and $D e=\frac{\dot{\gamma}_{0}}{D_{r}}$, for the shear rate $\dot{\gamma}_{0}=\frac{v_{0}}{h}$ and the effective viscous stress $\tau_{e f f}=\dot{\gamma}_{0} \eta_{0}$. In either case, $E r$ and $D e$ satisfy

$$
\frac{E r}{D e}=\frac{\eta_{0} D_{r}}{\tau_{F}}=\frac{t_{0}}{D_{r}^{-1}}=\frac{8 h^{2}}{N \mathcal{L}^{2}}=\alpha .
$$


While the Ericksen number measures the strength of distortional stress relative to the applied stress at the boundary, $\alpha$ represents the relative strengths of the excluded volume potential and the distortional elasticity potential, independent of the driving conditions. The nondimensional boundary conditions are

$$
\left\{\begin{array}{l}
\tau_{x y}\left(y= \pm \frac{1}{2}\right)=E r \cos \omega t, \quad \text { for imposed stress } \\
v_{x}\left(y= \pm \frac{1}{2}\right)= \pm E r \cos \omega t,
\end{array}\right.
$$

In the Appendix, we show that the solutions for these two rheometric boundary conditions are equivalent up to a rescaling and a phase shift. This calculations establishes that the moduli are independent of stress-controlled or velocity-controlled boundary conditions, which is not transparent as it is with isotropic linear viscoelastic constitutive laws. Thus, for the remainder of this paper, we choose to impose velocity boundary conditions.

The dimensionless evolution equation of $\mathbf{M}$ is given by [Wang, 2002]:

$$
\begin{aligned}
\frac{\partial}{\partial t} \mathbf{M}= & \boldsymbol{\Omega} \cdot \mathbf{M}-\mathbf{M} \cdot \mathbf{\Omega}+a\left(\mathbf{D} \cdot \mathbf{M}+\mathbf{M} \cdot \mathbf{D}-2 \mathbf{D}: \mathbf{M}_{4}\right) \\
& -6 \alpha\left[\mathbf{Q}-N\left(\mathbf{M} \cdot \mathbf{M}-\mathbf{M}: \mathbf{M}_{4}\right)\right]+\Delta \mathbf{M} \cdot \mathbf{M}+\mathbf{M} \cdot \Delta \mathbf{M}-2 \Delta \mathbf{M}: \mathbf{M}_{4},
\end{aligned}
$$

where $\mathbf{M}_{4}$ is the fourth moment of $f$, which we approximate by $\mathbf{M}_{4} \approx \mathbf{M M}$ to close the system on $\mathbf{M}$ and $\mathbf{v} .{ }^{1}$ The molecular shape parameter is $a=\frac{R^{2}-1}{R^{2}+1}$, where $R$ is the aspect ratio of the spheroidal molecules.

The Reynolds number for this nondimensionalization is $R e=\frac{\rho h^{2}}{\tau_{F} t_{0}^{2}}$, which we assume to be quite small, and so in the linear momentum balance we use the Stokes limit

$$
\mathbf{0}=\nabla \cdot \boldsymbol{\tau}
$$

The dimensionless extra stress tensor $\boldsymbol{\tau}$ from [Wang, 2002] can be written as the sum of a viscous stress and two types of elastic stress. The viscous stress is

$$
\boldsymbol{\tau}^{V i s}=\mu_{1}(\mathbf{D} \cdot \mathbf{M}+\mathbf{M} \cdot \mathbf{D})+\mu_{2} \mathbf{D}: \mathbf{M}_{4}+\mu_{3} \mathbf{D}
$$

where $\mu_{1}=\frac{3 \zeta_{1} D_{r}}{s_{0}^{2}}, \mu_{2}=\frac{3 \zeta_{2} D_{r}}{s_{0}^{2}}$, and $\mu_{3}=\frac{2 \eta_{s}}{\eta_{0}}+\frac{3 \zeta_{3} D_{r}}{s_{0}^{2}}$ for the solvent viscosity $\eta_{s}$ and the three shape-dependent friction coefficients $\zeta_{i}$ defined in [Wang, 2002]. The nematic elastic stress arises from being out of nematic equilibrium:

$$
\boldsymbol{\tau}^{N E}=\frac{3 a}{s_{0}^{2}} \alpha\left[\mathbf{Q}-N\left(\mathbf{M} \cdot \mathbf{M}-\mathbf{M}: \mathbf{M}_{4}\right)\right] .
$$

Note that at the nematic equilibrium $\boldsymbol{\tau}^{N E}\left(\mathbf{Q}_{0}\right)=\mathbf{0}$. The distortional elastic stress is

$$
\boldsymbol{\tau}^{D E}=\frac{1-a}{2 s_{0}^{2}} \mathbf{M} \cdot \Delta \mathbf{M}-\frac{1+a}{2 s_{0}^{2}} \Delta \mathbf{M} \cdot \mathbf{M}+\frac{a}{s_{0}^{2}} \Delta \mathbf{M}: \mathbf{M}_{4}-\frac{1}{4 s_{0}^{2}}\left(M_{k l, i} M_{k l, j}-\mathbf{M}: \nabla \nabla \mathbf{M}\right) .
$$

\footnotetext{
${ }^{1}$ The version of the model in [Choate et al., 2008] includes anisotropic distortional elasticity terms proportional to a parameter $\theta$ that are omitted here. The $\theta=0$ limit is analogous to the single Frank elastic constant approximation from LE theory. The inclusion of nonzero $\theta$ does not significantly change the results below, yet makes the model and discussion more complicated.
} 
We observe that $\boldsymbol{\tau}^{N E}$ is present in the monodomain model but absent in the LE model, which has only distortional elastic stresses.

The system we solve is for the primary velocity $v_{x}$, two order parameters $s$ and $\beta$, and the single director angle $\psi$ which determines the eigenvector frame of the orientation tensor as $\mathbf{n}_{1}=(\cos \psi, \sin \psi, 0), \mathbf{n}_{2}=(-\sin \psi, \cos \psi, 0)$, and $\mathbf{n}_{3}=(0,0,1)$. The boundary conditions for the orientation tensor correspond to the equilibrium uniaxial nematic phase, for the order parameters are fixed, $s\left(y= \pm \frac{1}{2}\right)=s_{0}, \beta\left(y= \pm \frac{1}{2}\right)=0$, while the principal orientation angle is arbitrary, $\psi\left(y= \pm \frac{1}{2}\right)=\psi_{0}$.

From (2), the small Ericksen number limit is the appropriate asymptotic limit to study small amplitude flow. We remind the reader that the following analysis will pay large dividends in that we will extract tractable model equations that we can solve semi-analytically and recover full scaling behavior of dynamic moduli. Without this analysis, one must solve the full system of partial differential equations across a multidimensional parameter space, then assimilate the results and try to glean scaling behavior. That task is essentially impossible. Instead, we employ the following solution ansatz for the orientation variables, flow velocity, and shear stress:

$$
\begin{aligned}
s & =s_{0}+\sum_{k=1}^{\infty} \operatorname{Er}^{k} s^{(k)}(y, t), \quad \beta=0+\sum_{k=1}^{\infty} \operatorname{Er}^{k} \beta^{(k)}(y, t), \\
\psi & =\psi_{0}+\sum_{k=1}^{\infty} \operatorname{Er}^{k} \psi^{(k)}(y, t), \quad v_{x}=0+\sum_{k=1}^{\infty} \operatorname{Er}^{k} v_{x}^{(k)}(y, t), \\
\tau_{x y} & =0+\sum_{k=1}^{\infty} \operatorname{Er}^{k} \tau_{x y}^{(k)}(t) .
\end{aligned}
$$

At $O(E r)$, the system (3) and (4) transforms to

$$
\begin{array}{rrr}
\frac{\partial s^{(1)}}{\partial t}= & A_{1} s^{(1)}+A_{2} \beta^{(1)}+A_{3} \frac{\partial^{2} s^{(1)}}{\partial y^{2}}+A_{4} \frac{\partial^{2} \beta^{(1)}}{\partial y^{2}} & +B_{1} \frac{\partial v_{x}^{(1)}}{\partial y}, \\
\frac{\partial \beta^{(1)}}{\partial t}= & +A_{6} \frac{\partial^{2} \beta^{(1)}}{\partial y^{2}} & +B_{2} \frac{\partial v_{x}^{(1)}}{\partial y}, \\
\frac{\partial \psi^{(1)}}{\partial t}= & C_{1} \frac{\partial^{2} \psi^{(1)}}{\partial y^{2}}+C_{2} \frac{\partial v_{x}^{(1)}}{\partial y}, \\
\tau_{x y}^{(1)}=B_{3} s^{(1)}+B_{4} \beta^{(1)}+B_{5} \frac{\partial^{2} s^{(1)}}{\partial y^{2}}+B_{6} \frac{\partial^{2} \beta^{(1)}}{\partial y^{2}}-C_{2} \frac{\partial^{2} \psi^{(1)}}{\partial y^{2}}+C_{3} \frac{\partial v_{x}^{(1)}}{\partial y}
\end{array}
$$

where

$$
\begin{gathered}
A_{1}=2 \alpha\left(6-N\left(s_{0}+2\right)\right), \quad A_{2}=4 \alpha N s_{0}\left(s_{0}-1\right), \quad A_{3}=\frac{2}{N}, \\
A_{4}=\frac{2 s_{0}\left(s_{0}-1\right)}{3}, \quad A_{5}=-6 \alpha N s_{0}, \quad A_{6}=\frac{2\left(1-s_{0}\right)}{3}, \\
B_{1}=\frac{a\left(1+2 s_{0}-3 s_{0}^{2}\right)}{3} \sin 2 \psi_{0}, B_{2}=\frac{a\left(s_{0}-1\right)}{3} \sin 2 \psi_{0}, B_{3}=-\frac{a A_{1}}{4 s_{0}^{2}} \sin 2 \psi_{0}, \\
B_{4}=\frac{a\left(A_{5}-A_{2}\right)}{4 s_{0}^{2}} \sin 2 \psi_{0}, B_{5}=-\frac{a A_{3}}{4 s_{0}^{2}} \sin 2 \psi_{0}, B_{6}=\frac{a\left(A_{6}-A_{4}\right)}{4 s_{0}^{2}} \sin 2 \psi_{0},
\end{gathered}
$$




$$
C_{1}=\frac{2+s_{0}}{3}, \quad C_{2}=-\frac{1}{2}\left(1-\lambda_{L} \cos 2 \psi_{0}\right), \quad C_{3}=\frac{\mu_{1}\left(s_{0}+2\right)}{6}+\frac{\mu_{2} s_{0}^{2}}{4} \sin ^{2} 2 \psi_{0}+\frac{\mu_{3}}{2},
$$

for the Leslie tumbling parameter $\lambda_{L}=\frac{a\left(2+s_{0}\right)}{3 s_{0}}$ [Forest and Wang, 2003].

The coefficients $B_{i}$ are odd functions of $\psi_{0}$ whereas the coefficients $A_{i}$ and $C_{i}$ are even functions of $\psi_{0}$, which leads to the following symmetry: if $\left\{s^{(1)}, \beta^{(1)}, \psi^{(1)}, v_{x}^{(1)}\right\}$ is a solution for $\psi_{0}$, then the solution corresponding to $-\psi_{0}$ is $\left\{-s^{(1)},-\beta^{(1)}, \psi^{(1)}, v_{x}^{(1)}\right\}$. Furthermore, since $G^{\prime}(\omega)$ and $G^{\prime \prime}(\omega)$ depend only on the in- and out-of-phase components of $\tau_{x y}^{(1)}$ and $v_{x}^{(1)}$, the moduli are even functions of $\psi_{0}$. Therefore, we only consider the values $0 \leq \psi_{0} \leq \frac{\pi}{2}$.

Since we have the sinusoidal driving conditions (2) and our end goal is to find the dynamic moduli, we assume the phased-locked solutions

$$
\begin{aligned}
& s^{(1)}(y, t)=s_{1}(y) \cos \omega t+s_{2}(y) \sin \omega t, \quad \beta^{(1)}(y, t)=\beta_{1}(y) \cos \omega t+\beta_{2}(y) \sin \omega t, \\
& \psi^{(1)}(y, t)=\psi_{1}(y) \cos \omega t+\psi_{2}(y) \sin \omega t, \quad v_{x}^{(1)}(y, t)=v_{1}(y) \cos \omega t+v_{2}(y) \sin \omega t,
\end{aligned}
$$

and the phased-locked stress $\tau_{x y}^{(1)}(t)=\tau_{1} \cos \omega t+\tau_{2} \sin \omega t=\frac{2}{\omega}\left[G^{\prime \prime}(\omega) \cos \omega t+G^{\prime}(\omega) \sin \omega t\right]$.

\section{Simplifications of the model}

Imbedded within the full system (9)-(13) are two simpler and complimentary models that are analytically solvable. If we ignore the order parameter terms, we recover the Leslie-Ericksen equations, and if we ignore any spatial gradients in the orientational variables and assume a linear velocity profile, we recover the monodomain equations. We recall the explicit moduli predictions of these two limits now because we will find that the full response for oblique anchoring is essentially a superposition of these simplified limits.

\subsection{Leslie-Ericksen with oblique anchoring}

In [Choate et al., 2008], we observed that the coefficients $B_{i}$ were all proportional to $\sin 2 \psi_{0}$ so that if we chose either $\psi_{0}=0$ or $\frac{\pi}{2}$, we could decouple the system (9)-(13) into two systems, a trivial order parameter system and a director angle-velocity system that takes the form similar to Leslie-Ericksen theory:

$$
\begin{array}{rlr}
\frac{\partial \psi_{L E}^{(1)}}{\partial t}= & C_{1} \frac{\partial^{2} \psi_{L E}^{(1)}}{\partial y^{2}}+C_{2} \frac{\partial v_{L E}^{(1)}}{\partial y} \\
\tau_{L E}^{(1)}= & -C_{2} \frac{\partial^{2} \psi_{L E}^{(1)}}{\partial y^{2}}+C_{3} \frac{\partial v_{L E}^{(1)}}{\partial y} \\
0=\frac{\partial \tau_{L E}^{(1)}}{\partial y} &
\end{array}
$$

In terms of the LE parameters, the coefficients are $C_{1}=\frac{\eta_{1}}{\gamma_{1}}, C_{2}=-\frac{1}{2}\left(1-\lambda_{L} \cos 2 \psi_{0}\right)$, and $C_{3}=1-\frac{\alpha_{2}^{2}}{\eta_{1} \gamma_{1}}+\left(\frac{\alpha_{1}-\lambda_{L} \gamma_{2}}{4 \eta_{1}}\right) \sin ^{2} 2 \psi_{0}$, which exhibit the same functional dependence on $\psi_{0}$ as (16). This gives the stress-strain relationship needed to identify the storage and loss moduli 
as, respectively,

$$
\begin{aligned}
& G_{L E}^{\prime}(\omega)=\frac{2 C_{2}^{2} C_{3}^{2} \omega^{2} r(\sinh r-\sin r)}{\left(8 C_{2}^{4} r^{2}+C_{3}^{2} \omega^{2}\right)(\cosh r+\cos r)-16 C_{2}^{4} r^{2} \cos r-4 C_{2}^{2} C_{3} r \omega(\sin r+\sinh r)}, \\
& G_{L E}^{\prime \prime}(\omega)=\frac{C_{3}^{2} \omega^{2}\left(C_{3} \omega(\cosh r+\cos r)-2 C_{2}^{2} r(\sinh r+\sin r)\right)}{\left(8 C_{2}^{4} r^{2}+C_{3}^{2} \omega^{2}\right)(\cosh r+\cos r)-16 C_{2}^{4} r^{2} \cos r-4 C_{2}^{2} C_{3} r \omega(\sin r+\sinh r)},
\end{aligned}
$$

for $r=\sqrt{\frac{C_{3} \omega}{2\left(C_{1} C_{3}+C_{2}^{2}\right)}}$.

It is interesting to note the consequences of selecting the Leslie alignment angle $\psi_{L}=$ $\frac{1}{2} \cos ^{-1} \frac{1}{\lambda_{L}}\left(\right.$ or $\left.-\psi_{L}\right)$ as the anchoring angle for nematics that flow-align under steady shear. In this case, $C_{2}=0$ so that $G_{L E}^{\prime}(\omega)=0$ and $G_{L E}^{\prime \prime}(\omega)=C_{3} \omega$. This is a generalization of the observation of [de Andrade Lima and Rey, 2004] for a $\lambda_{L}=1$ fluid with tangential anchoring.

The asymptotic behavior of the moduli for low and high frequencies are

$$
\begin{array}{lll}
G_{L E}^{\prime}(\omega)=\frac{C_{2}^{2}}{12 C_{1}^{2}} \omega^{2}, & G_{L E}^{\prime \prime}(\omega)=\left(C_{3}+\frac{C_{2}^{2}}{C_{1}}\right) \omega, & \text { as } \omega \rightarrow 0, \\
G_{L E}^{\prime}(\omega)=C_{2}^{2} \sqrt{\frac{2 C_{3}}{C_{1} C_{3}+C_{2}^{2}}} \sqrt{\omega}, & G_{L E}^{\prime \prime}(\omega)=C_{3} \omega, & \text { as } \omega \rightarrow \infty .
\end{array}
$$

\subsection{Monodomains with oblique anchoring}

In [Choate and Forest, 2006], we examined the monodomain problem in which it is assumed that $s^{(1)}, \beta^{(1)}$ and $\psi^{(1)}$ have no spatial gradients and that $v_{x}^{(1)}=2 y \cos \omega t$. Under these restrictions, we again see a decoupling of the full system, but now when $\sin 2 \psi_{0} \neq 0$, it is the order parameter system that is nontrivial:

$$
\begin{array}{ccc}
\frac{d s_{M D}^{(1)}}{d t}=A_{1} s_{M D}^{(1)}+A_{2} \beta_{M D}^{(1)} & +2 B_{1} \cos \omega t \\
\frac{d \beta_{M D}^{(1)}}{d t}= & A_{5} \beta_{M D}^{(1)} & +2 B_{2} \cos \omega t \\
\tau_{M D}^{(1)}=B_{3} s_{M D}^{(1)}+B_{4} \beta_{M D}^{(1)} & +2 C_{3} \cos \omega t
\end{array}
$$

From the solution to this system, the dynamic moduli can be written as

$$
\begin{aligned}
& G_{M D}^{\prime}(\omega)=\omega^{2}\left(\frac{B_{2}\left(B_{3}+2 B_{4}\right)}{2\left(A_{5}^{2}+\omega^{2}\right)}-\frac{B_{3}\left(B_{2}-2 B_{1}\right)}{2\left(A_{1}^{2}+\omega^{2}\right)}\right), \\
& G_{M D}^{\prime \prime}(\omega)=\omega\left(C_{3}-\frac{A_{5} B_{2}\left(B_{3}+2 B_{4}\right)}{2\left(A_{5}^{2}+\omega^{2}\right)}+\frac{A_{1} B_{3}\left(B_{2}-2 B_{1}\right)}{2\left(A_{1}^{2}+\omega^{2}\right)}\right) .
\end{aligned}
$$

It is interesting to note that $G_{M D}^{\prime}$ and $G_{M D}^{\prime \prime}$ have the same values for $\psi_{0}$ and $\frac{\pi}{2}-\psi_{0}$, but the LE predictions (19) do not share this symmetry. The monodomain asymptotic scaling behavior is

$$
\begin{aligned}
& G_{M D}^{\prime}(\omega)=\left(\frac{B_{2}\left(B_{3}+2 B_{4}\right)}{2 A_{5}^{2}}-\frac{B_{3}\left(B_{2}-2 B_{1}\right)}{2 A_{1}^{2}}\right) \omega^{2}, \\
& G_{M D}^{\prime \prime}(\omega)=\left(C_{3}-\frac{B_{2}\left(B_{3}+2 B_{4}\right)}{2 A_{5}}+\frac{B_{3}\left(B_{2}-2 B_{1}\right)}{2 A_{1}}\right) \omega, \quad \text { as } \omega \rightarrow 0, \\
& G_{M D}^{\prime}(\omega)=B_{2} B_{4}+B_{1} B_{3}, \quad G_{M D}^{\prime \prime}(\omega)=C_{3} \omega, \quad \text { as } \omega \rightarrow \infty .
\end{aligned}
$$




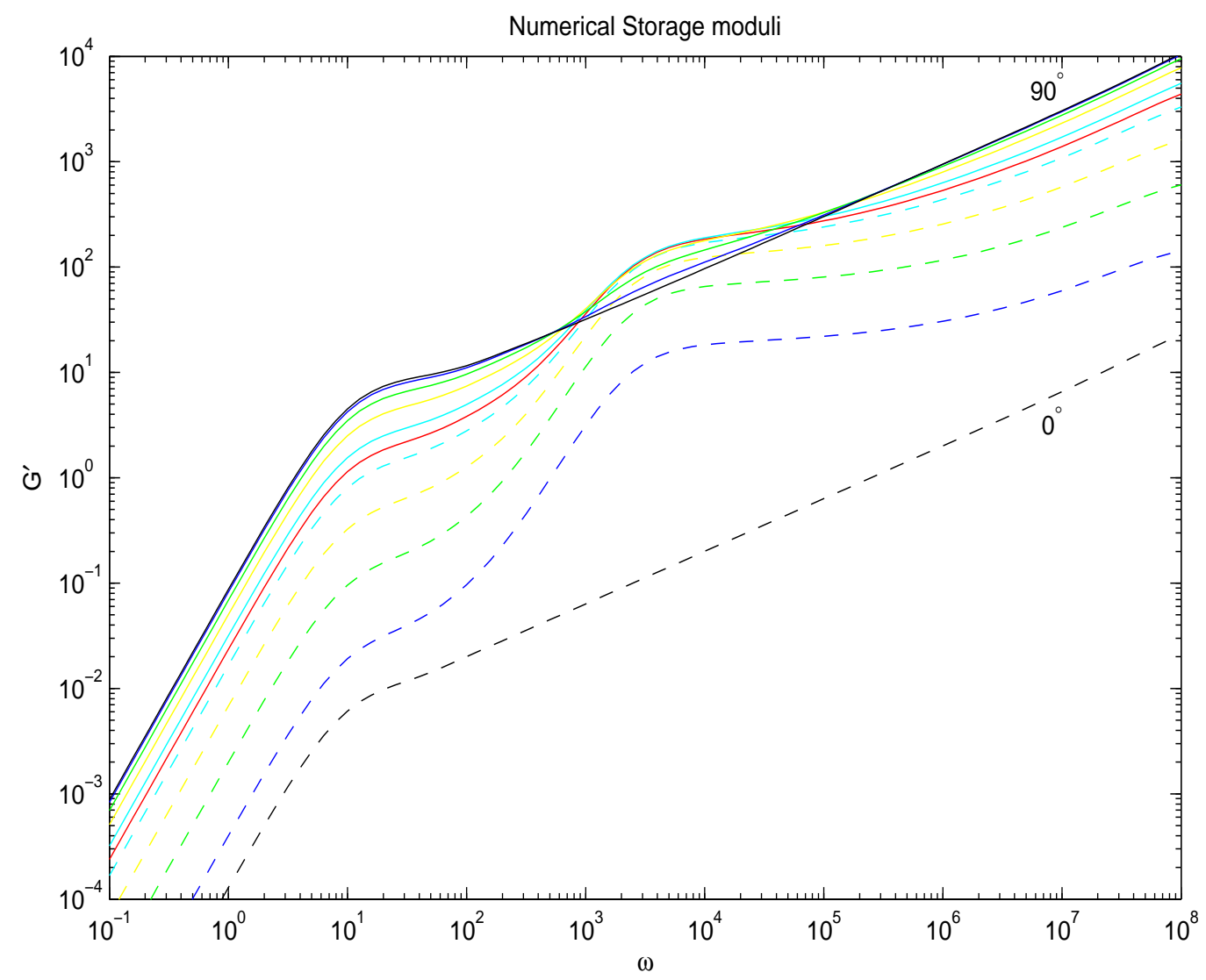

Figure 1: The storage modulus $G^{\prime}(\omega)$ for anchoring angles $\psi_{0}=0^{\circ}, 10^{\circ}, 20^{\circ}, 30^{\circ}, 40^{\circ}, 45^{\circ}$, $50^{\circ}, 60^{\circ}, 70^{\circ}, 80^{\circ}$, and $90^{\circ}$.

\section{Numerical solutions}

We now return to the full system for nematic polymers with oblique anchoring angles (9)(13) and solve the model equations numerically. Using the in-phase $\tau_{1}$ and out-of-phase $\tau_{2}$ components of the shear stress, we can define the dynamic moduli as

$$
G^{\prime}(\omega)=\frac{\omega}{2} \tau_{2}, \quad G^{\prime \prime}(\omega)=\frac{\omega}{2} \tau_{1}
$$

\subsection{Storage modulus}

Figure 1 depicts the complicated dependence of the storage modulus $G^{\prime}(\omega)$ on the anchoring angle. For low frequencies, $G^{\prime}(\omega)$ scales like $\omega^{2}$ until reaching a plateau for moderate frequencies. For higher frequencies, when $\psi_{0}$ is 0 or $\frac{\pi}{2}, G^{\prime}(\omega)$ follows the LE prediction and scales like $\sqrt{\omega}$. However, for $\psi_{0}$ near $\frac{\pi}{4}, G^{\prime}(\omega)$ increases more rapidly to a secondary plateau for a range of frequencies. The maximum $G^{\prime}$ is for an anchoring angle near $50^{\circ}$. After this secondary plateau, all anchoring angles scale like $\sqrt{\omega}$, and their dependence on $\psi_{0}$ is similar to that for low frequencies. 
The effect of the anchoring angle is complicated by the molecular shape parameter $a$. The nematic shown in Figure 1 tumbles in response to a steady shear flow $\left(\left|\lambda_{L}\right|<1\right)$. Figure 2 shows $G^{\prime}$ as a function of $\psi_{0}$ for two frequencies for this same value of $\lambda_{L}$, but it also adds a steady-shear flow-aligning nematic $\left(\left|\lambda_{L}\right|>1\right)$ for contrast. In the tumbling case, $G^{\prime}$ increases monotonically with $\psi_{0}$ for low frequencies. However, we observed earlier that the LE model predicts that $G_{L E}^{\prime}(\omega) \equiv 0$ for all frequencies if the Leslie angle $\psi_{L}$ was chosen as the anchoring angle. We see now that for low frequencies, $G^{\prime}(\omega)$ is nonzero for all $\psi_{0}$, but attains its minimum and is orders of magnitude smaller when $\psi_{0}$ is near $\psi_{L}$. However in the secondary plateau for moderate frequencies, this behavior is not observed, and steady-shear flow-aligning and tumbling nematics behave very similarly with a rapid increase with $\psi_{0}$ from the minimum value at tangential anchoring.

We now observe that we can accurately capture the behavior of the full solution with a hybrid model consisting of a linear superposition of the Leslie-Ericksen prediction and the monodomain prediction:

$$
G_{h y b}^{\prime}=G_{L E}^{\prime}+G_{M D}^{\prime}
$$

Figure 3 compares the prominent features of the LE and monodomain models with the numerical solution to show why the hybrid model is successful. For low frequencies, the monodomain prediction is orders of magnitude smaller than the LE prediction because the bulk nematic elastic stress in negligible compared to the distortional elastic stress.

However, as the LE prediction plateaus and the distortional elastic stress enters the $O(\sqrt{\omega})$ regime, the monodomain contribution of the nematic elastic stress continues $O\left(\omega^{2}\right)$ growth until it catches and surpasses the LE contribution. This coincides with the secondary plateau for the full system. Since the monodomain prediction levels off to $O(1)$ behavior, the $O(\sqrt{\omega})$ LE contribution eventually regains dominance for high frequencies. This hybrid is not perfect though. While it appears that the numerical prediction is $O(\sqrt{\omega})$ as $\omega \rightarrow \infty$ as is $G_{h y b}^{\prime}$, the numerical solution is slightly larger than the hybrid prediction.

Also, we find that the sum of the monodomain prediction's dependence on $\psi_{0}$ through direct proportionality to $\sin ^{2} 2 \psi_{0}$ and the more complicated dependence of the LE prediction effectively mimics the full system's anchoring angle dependence.

\subsection{Loss modulus}

The loss modulus $G^{\prime \prime}(\omega)$ is mostly linear with $\omega$, and so we choose to analyze it through the frequency-dependent viscosity $\eta^{\prime}(\omega)=\frac{G^{\prime \prime}(\omega)}{\omega}$. Figure 4 shows values of $\eta^{\prime}(\omega)$ for several different values of $\psi_{0}$ between 0 and $90^{\circ}$. We find three different plateaus which decrease from low to intermediate to high frequencies although for some anchoring angles, these three states are nearly indistinguishable. Near tangential anchoring, the viscosity shows very little frequency dependence. Near normal anchoring, there is a steep falloff from the low frequency regime to a poorly defined intermediate regime that slowly settles to the high frequency limit. However, for anchoring angles between $30^{\circ}$ and $60^{\circ}$, we do observe three distinct regimes.

Notice that the high frequency limit has the symmetry $\eta^{\prime}\left(\psi_{0}\right)=\eta^{\prime}\left(\frac{\pi}{2}-\psi_{0}\right)$, with the maximum value occuring for $\psi_{0}=\frac{\pi}{4}$. From (20) and (23), this symmetry is also present in both the Leslie-Ericksen and monodomain limits: $\eta_{L E}^{\prime} \rightarrow C_{3}=\frac{\mu_{1}\left(s_{0}+2\right)}{6}+\frac{\mu_{2} s_{0}^{2}}{4} \sin ^{2} 2 \psi_{0}+\frac{\mu_{3}}{2}$ 

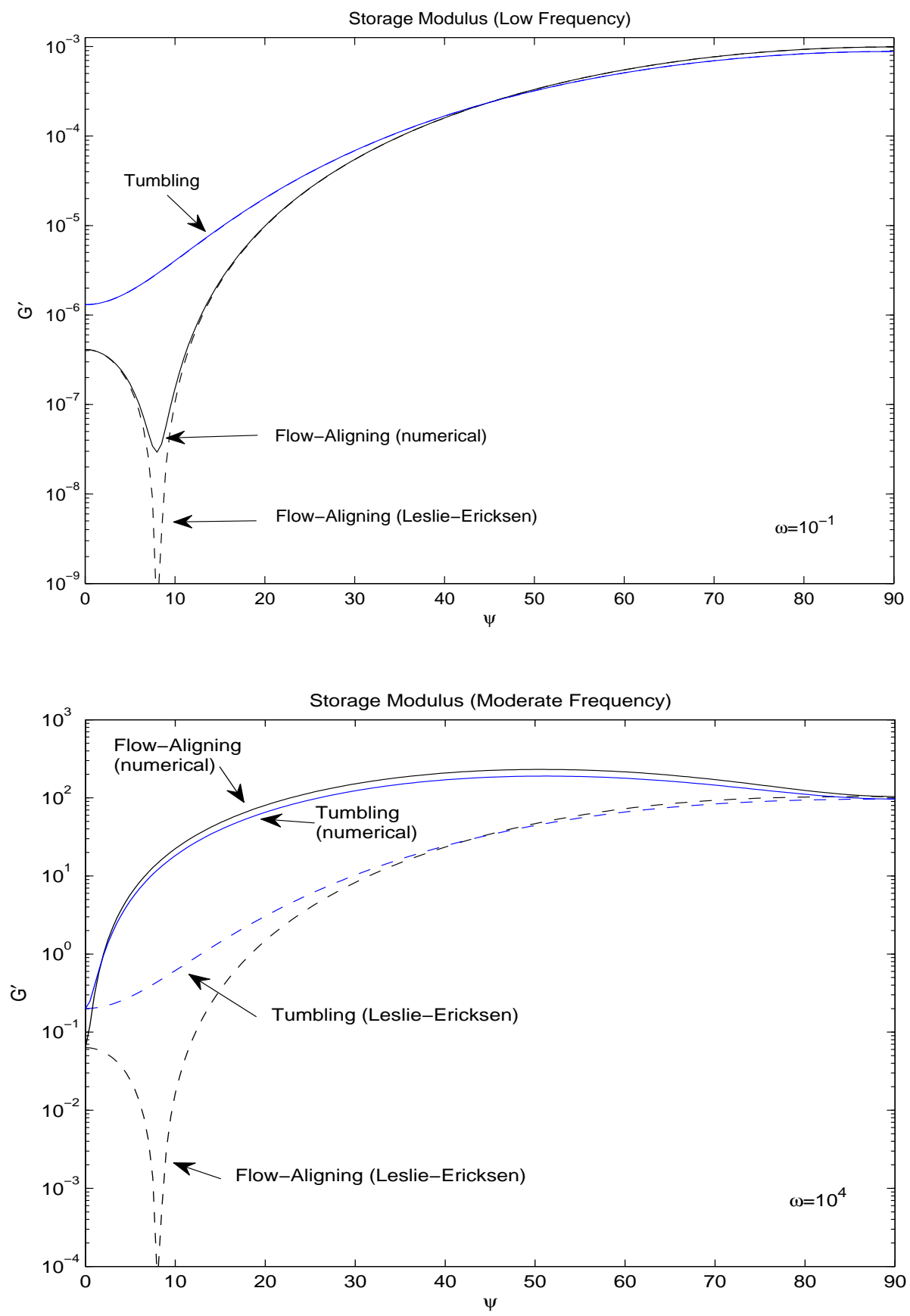

Figure 2: The effect of the molecular shape on $G^{\prime}$. For low frequencies, a steady-shear flow-aligning nematic has its minimum when $\psi_{0}=\psi_{L}$. The dashed curve shows the LE prediction, which is zero when $\psi_{0}=\psi_{L}$. This minimum is not present for steady-shear tumbling nematics. However, for higher frequencies, the nematic elastic stress contribution becomes strong enough to counteract this minimum which is still present in the LE prediction. 


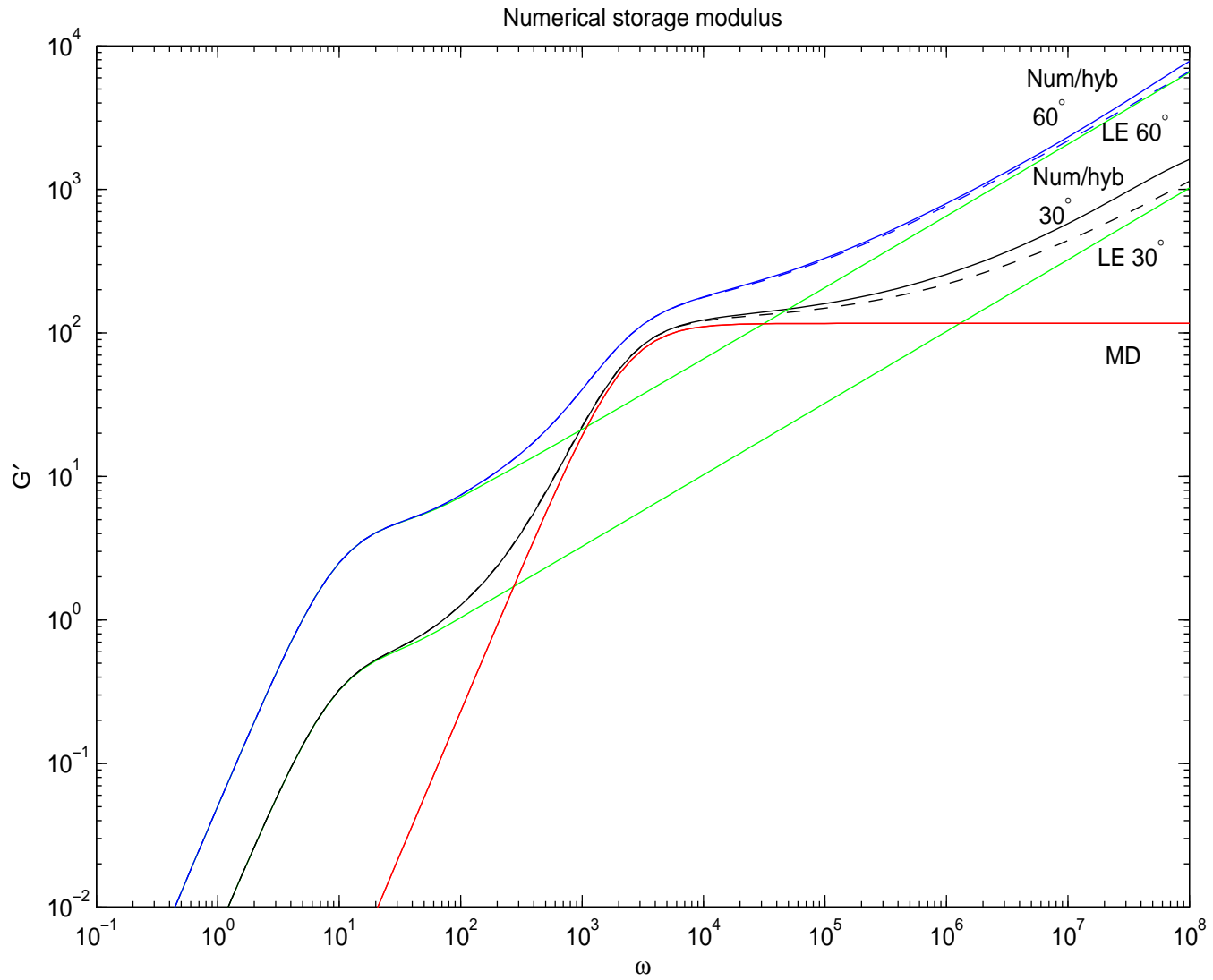

Figure 3: The storage modulus $G^{\prime}(\omega)$ for $\psi_{0}=30^{\circ}$ (black) and $60^{\circ}$ (blue). The LeslieEricksen prediction is green, and the monodomain prediction is red. Notice that $G_{M D}^{\prime}\left(\frac{\pi}{2}-\right.$ $\left.\psi_{0}\right)=G_{M D}^{\prime}\left(\psi_{0}\right)$, but the LE predition does not share this symmetry. The dotted curves are the hybrid models (the sum of the red and green curves) and are indistinguishable from the solid numerical curves for low and moderate frequencies. 


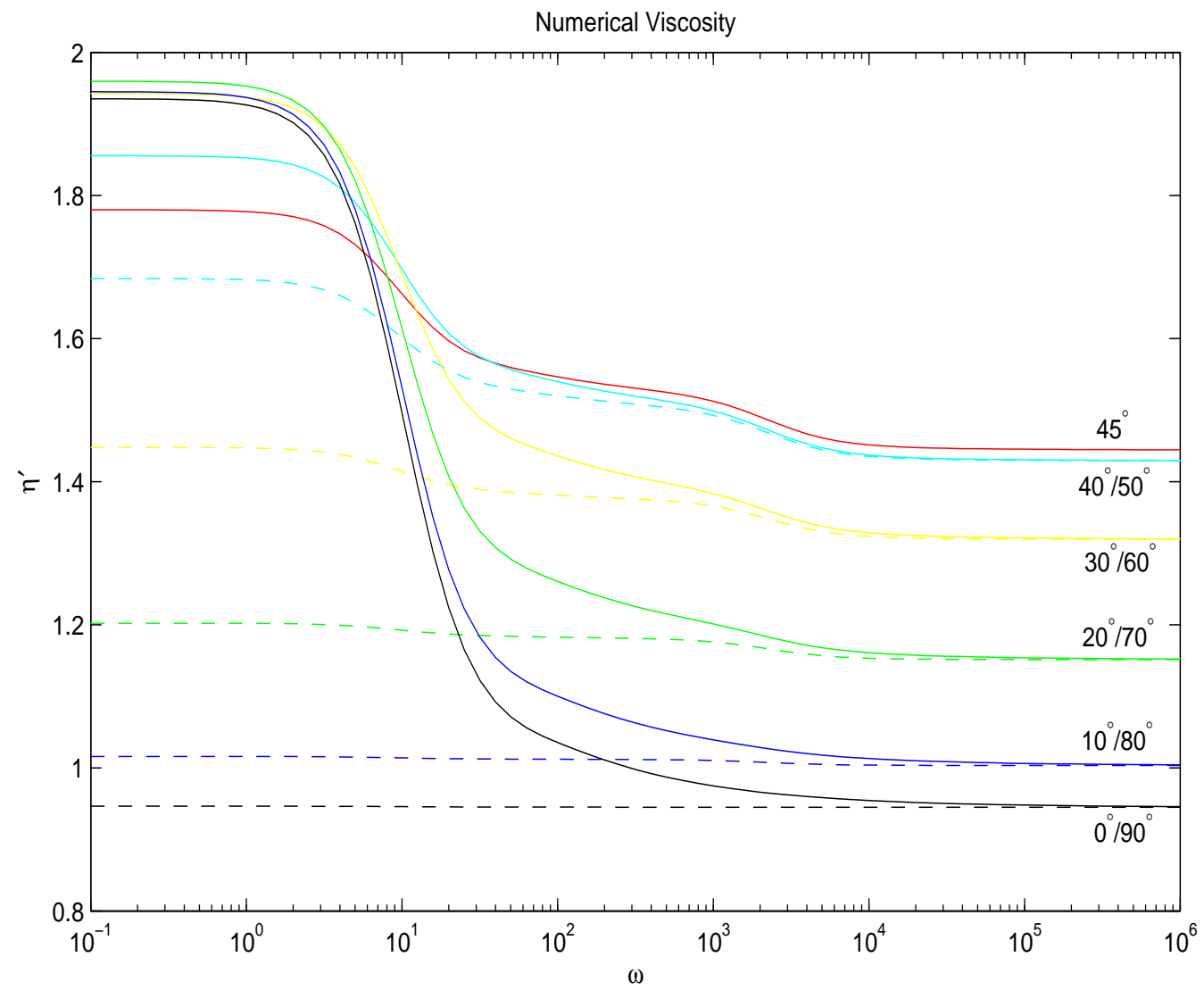

Figure 4: The frequency-dependent viscosity $\eta^{\prime}(\omega)=\frac{G^{\prime \prime}(\omega)}{\omega}$ for the anchoring angles $\psi_{0}=0^{\circ}$, $10^{\circ}, 20^{\circ}, 30^{\circ}, 40^{\circ}, 45^{\circ}, 50^{\circ}, 60^{\circ}, 70^{\circ}, 80^{\circ}, 90^{\circ}$. 
and $\eta_{M D}^{\prime} \rightarrow C_{3}$ as $\omega \rightarrow \infty$. The low frequency behavior does not share this symmetry, but instead has a maximum near $\psi_{0} \approx 65^{\circ}$.

Figure 5 shows more detail of the $\psi_{0}$ dependence and also highlights the effect of the shape parameter. For low frequencies, steady-shear flow-aligning nematics are more viscous than tumbling nematics except near tangential anchoring, and the maximum occurs at a shape-dependent angle in between $50^{\circ}$ and $70^{\circ}$. At intermediate and higher frequencies, the director angle symmetry emerges, and we also see that near normal anchoring, the viscosity of steady-shear tumbling nematics becomes slightly more viscous than flow-aligning.

Again, we found a very good approximation of the numerical $\eta^{\prime}$ with a hybrid model with a linear superposition of the Leslie Ericksen and monodomain model, but with a necessary translation factor $C_{3}$ :

$$
\eta_{h y b}^{\prime}=\eta_{L E}^{\prime}+\eta_{M D}^{\prime}-C_{3}
$$

Figure 6 shows that $\eta_{h y b}^{\prime}$ is practically indistinguishable from the numerical solution with a relative error of less than $0.1 \%$ for all frequencies.

The reason for the form (26) can be seen by rewriting the shear stress (12) as

$$
\begin{aligned}
\tau_{x y}^{(1)}=[ & \left.B_{3} s^{(1)}+B_{4} \beta^{(1)}+2 C_{3} \cos \omega t\right]-2 C_{3} \cos \omega t \\
& +B_{5} \frac{\partial^{2} s^{(1)}}{\partial y^{2}}+B_{6} \frac{\partial^{2} \beta^{(1)}}{\partial y^{2}}+\left(-C_{2} \frac{\partial^{2} \psi^{(1)}}{\partial y^{2}}+C_{3} \frac{\partial v_{x}^{(1)}}{\partial y}\right) .
\end{aligned}
$$

The terms in square brackets take the same basic form as the monodomain stress (21) while the terms in parentheses take the same basic form as the LE stress (18). The $-2 C_{3} \cos \omega t$ term leads to the $-C_{3}$ term in (26), and since it is in-phase with the plates under our imposed velocity boundary conditions, it makes no contribution to the storage modulus $G_{h y p}^{\prime}(\omega)$.

\section{The role of the gap width}

The dimensional gap width $h$ enters into both the characteristic stress $\tau_{F}=\frac{\nu k T N \mathcal{L}^{2} s_{0}^{2}}{8 h^{2}}$ and characteristic time $t_{0}=\frac{8 h^{2}}{N \mathcal{L}^{2} D_{r}}$, but it does not appear in the characteristic viscosity $\eta_{0}=$ $\frac{\nu k T s_{0}^{2}}{D_{r}}$. The only nondimensional parameter in the coefficients (14)-(16) in which $h$ appears is $\alpha=\frac{8 h^{2}}{N \mathcal{L}^{2}}=\frac{E r}{D e}$. Since the characteristic time and stress were chosen to draw comparisons with Leslie-Ericksen theory, $\alpha$ does not appear in the Leslie-Ericksen system (18), and the nondimensional $G_{L E}^{\prime}(\omega)$ and $G_{L E}^{\prime \prime}(\omega)$ are independent of $\alpha$ and therefore the gap width.

In contrast, the monodomain moduli predictions do depend on the gap width under this nondimensionalization. Both the monodomain storage and loss moduli satisfy the relationship

$$
G_{M D}(\omega ; \alpha)=\alpha G_{M D}\left(\frac{\omega}{\alpha} ; 1\right) .
$$

This implies $\eta_{M D}(\omega, \alpha)=\eta_{M D}\left(\frac{\omega}{\alpha} ; 1\right)$.

Figure 7 shows the storage modulus and viscosity for different values of $\alpha$. If $\alpha$ is small, the secondary plateau is small, and the storage modulus is very similar to the LE prediction 

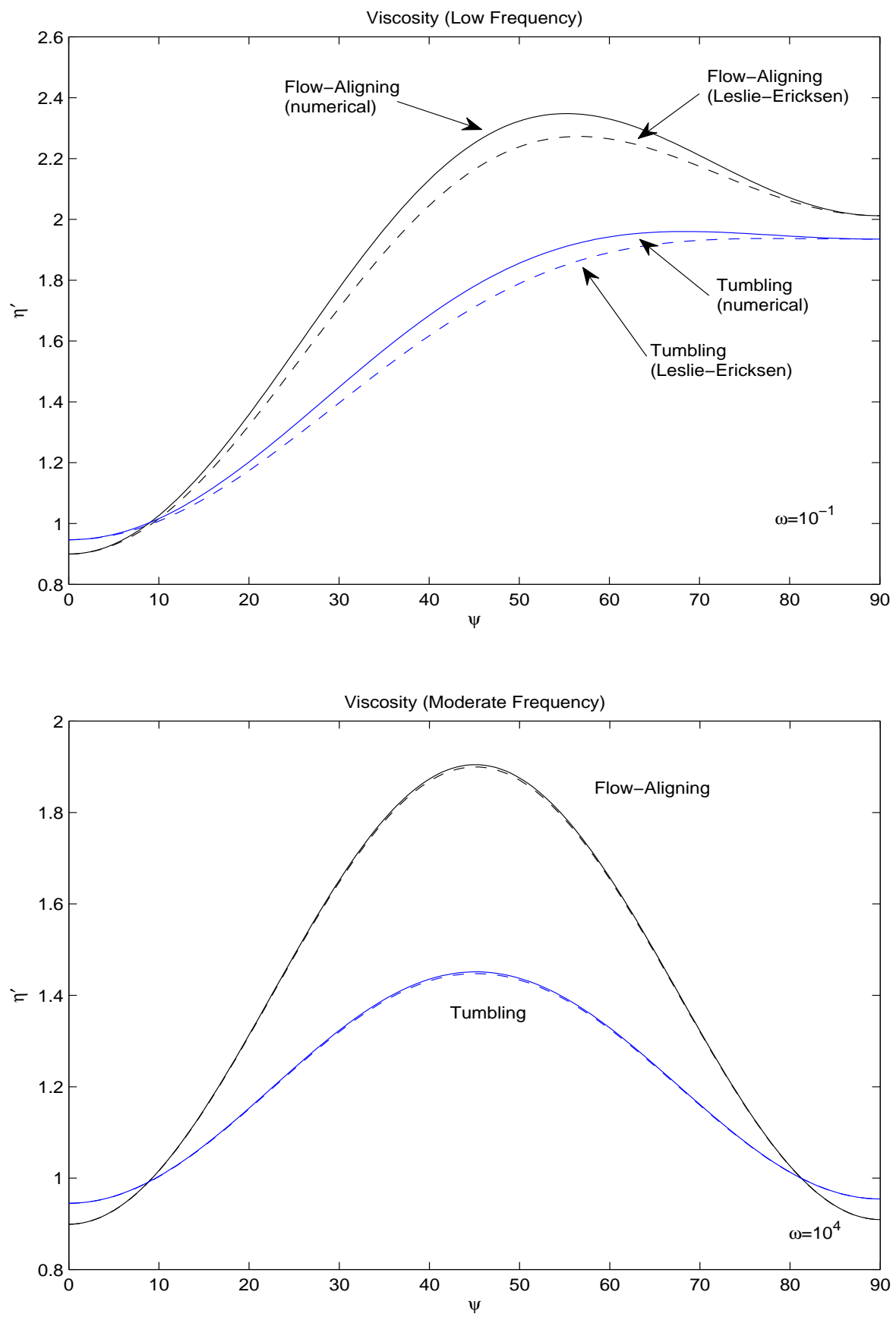

Figure 5: The effect of the molecular shape on $\eta^{\prime}$. For low frequencies, the maximum viscosity occurs for $\psi_{0}$ in between $50^{\circ}$ and $70^{\circ}$, depending on the shape parameter. For higher frequencies, the numerical, LE, monodomain, and hybrid models all approach the same limit $\eta^{\prime}=C_{3}$, which has the $\eta^{\prime}\left(\psi_{0}\right)=\eta^{\prime}\left(\frac{\pi}{2}-\psi_{0}\right)$ symmetry. 


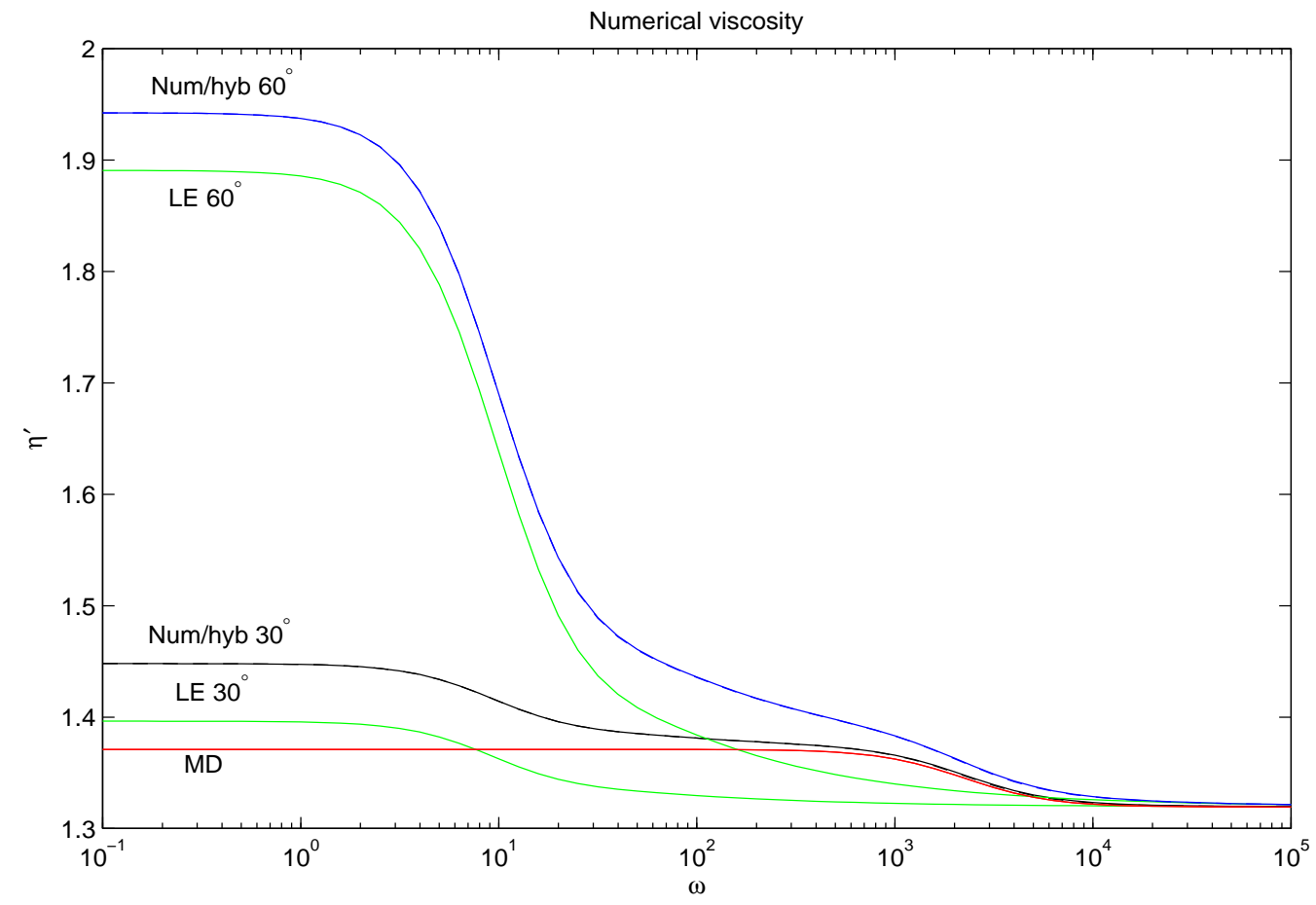

Figure 6: The viscosity $\eta^{\prime}=\frac{G^{\prime \prime}(\omega)}{\omega}$ for $\psi_{0}=30^{\circ}$ (black) and $60^{\circ}$ (blue). The dotted curves are the hybrid models and are indistinguishable from the numerical curve. The Leslie-Ericksen prediction is green, and the monodomain prediction is red. Notice that $\eta_{M D}^{\prime}\left(\frac{\pi}{2}-\psi_{0}\right)=$ $\eta_{M D}^{\prime}\left(\psi_{0}\right)$, but the LE version does not share this symmetry. 

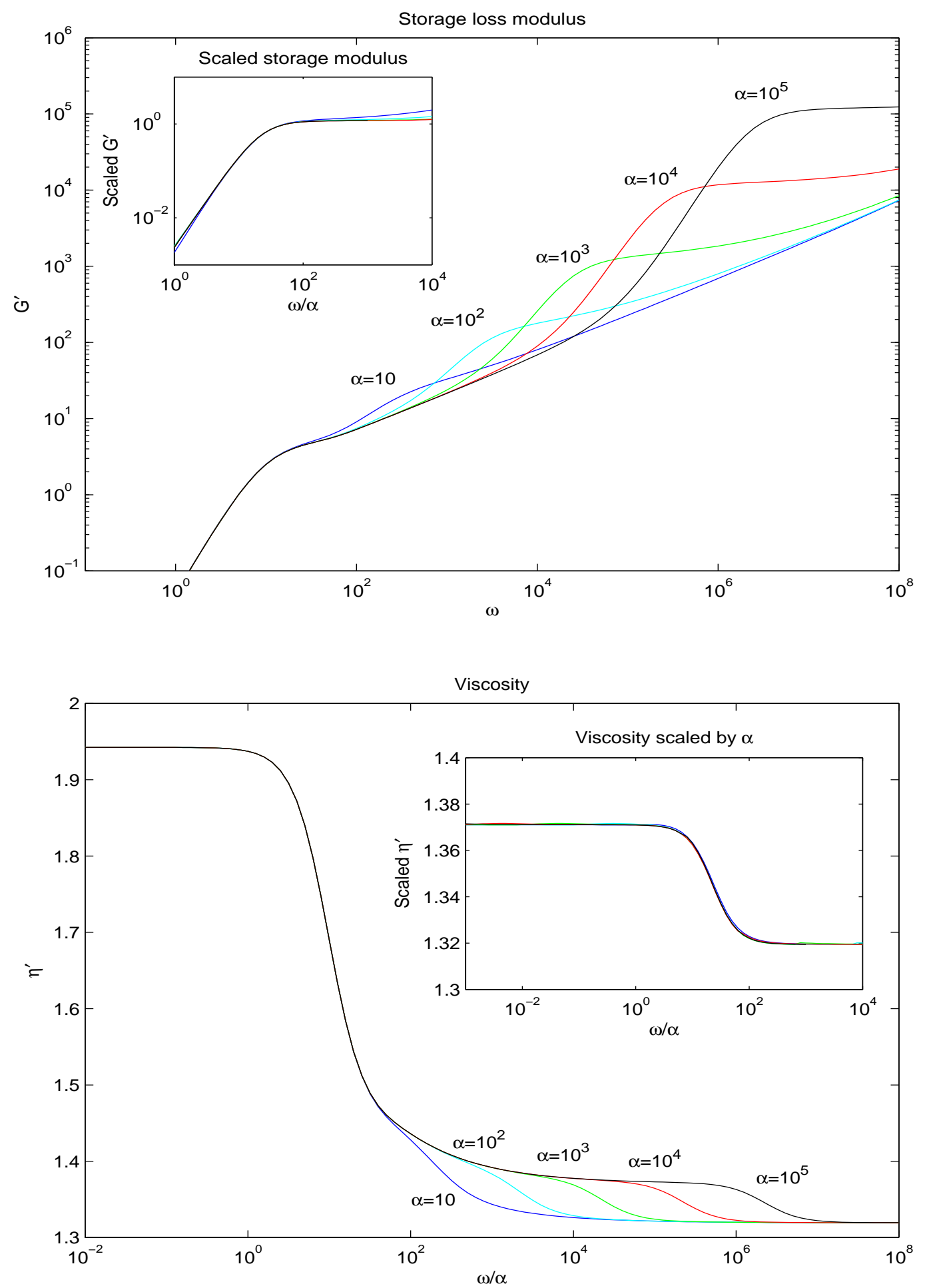

Figure 7: The storage modulus and viscosity for several values of $\alpha$ and $\psi_{0}=60^{\circ}$. 
although in the limit $\alpha \rightarrow 0$, we do not exactly recover the LE model. As $\alpha$ increases, the size of the secondary plateau increases, and its onset occurs at a larger frequency. For the viscosity, the values of the three regimes and the transition from the low to intermediate regimes are independent of $\alpha$. However, transition frequency from the intermediate to high frequency regimes increases with $\alpha$.

Since the monodomain contributions are zero for tangential and normal anchoring, it is only for oblique anchoring that this gap-width effect is observable.

Motivated by the hybrid models, we observe the following scalings. If we subtract the LE prediction, the remaining parts approximately collapse onto the monodomain prediction as shown in the inserts in Figure 7:

$$
\begin{aligned}
\frac{G^{\prime}(\omega ; \alpha)-G_{L E}^{\prime}(\omega)}{\alpha} & \approx G_{M D}^{\prime}\left(\frac{\omega}{\alpha} ; 1\right), \\
\eta^{\prime}(\omega ; \alpha)+C_{3}-\eta_{L E}^{\prime}(\omega) & \approx \eta_{M D}^{\prime}\left(\frac{\omega}{\alpha} ; 1\right) .
\end{aligned}
$$

\section{Conclusion}

We have analyzed the dynamic moduli of heterogeneous nematic polymers for anchoring conditions ranging from tangential to oblique to normal with respect to the plates. The asymptotic equations for the linear viscoelastic response consist of a four-dimensional system for the flow and orientational tensor, which we solve numerically. The results are then compared with previous results for monodomains [Choate and Forest, 2006] and for heterogeneous responses with normal and tangential anchoring [Choate et al., 2008], which are exactly solvable and scaling behavior of storage and loss moduli across the frequency spectrum is explicit.

We find a remarkable superposition principle for the dynamic moduli associated with oblique anchoring conditions. For both the storage and loss moduli, we find that for low and high frequencies, the distortional elastic stress is dominant indicating Leslie-Ericksenlike (or small molecule, liquid crystal like) behavior with relatively negligible contributions to $G^{\prime}(\omega)$ and $G^{\prime \prime}(\omega)$ from the monodomain bulk elastic stress. However, there is a window of moderate frequencies for which the monodomain elastic stresses are stronger than the distortional stresses for oblique anchoring angles. These results predict that the intermediate frequency range is where dynamic moduli are more sensitive to gap height, excluded volume interactions among the rod macromolecules, and relative value of Frank elastic constants.

\section{Acknowledgements}

The authors acknowledge research support in part by grants from AFOSR FA9550-06-1-0063, NSF DMS 0502266, 0604891, 0908423, and ARO W911NF-09-1-0389. 


\section{Appendix: Equivalence of stress and velocity controlled boundary conditions}

To see that the phased-locked solutions of (9)-(13) with imposed velocity and imposed stress boundary conditions are equivalent up to a rescaling and a phase shift, first denote the imposed velocity solution with a subscript $v: s_{v}^{(1)}, \quad \beta_{v}^{(1)}, \quad v_{x, v}^{(1)}, \quad \psi_{v}^{(1)}$. Associated with this solution is the resulting shear stress $\tau_{x y, v}^{(1)}(t)=\tau_{1, v} \cos \omega t+\tau_{2, v} \sin \omega t$. We define the quantities $E=\frac{1}{\sqrt{\tau_{1, v}^{2}+\tau_{2, v}^{2}}}$ and $\chi=-\tan ^{-1} \frac{\tau_{2, v}}{\tau_{1, v}}$. Note that $\sin \chi=-E \tau_{2, v}$ and $\cos \chi=E \tau_{1, v}$.

Now define the functions

$$
\begin{aligned}
s_{\tau}^{(1)}(y, t)=E s_{v}^{(1)}\left(y, t-\frac{\chi}{\omega}\right), & \beta_{\tau}^{(1)}(y, t)=E \beta_{v}^{(1)}\left(y, t-\frac{\chi}{\omega}\right), \\
v_{x, \tau}^{(1)}(y, t)=E v_{x, v}^{(1)}\left(y, t-\frac{\chi}{\omega}\right), & \psi_{\tau}^{(1)}(y, t)=E \psi_{v}^{(1)}\left(y, t-\frac{\chi}{\omega}\right) .
\end{aligned}
$$

These functions also define a general solution to the system (9)-(13). Associated with this solution is the shear stress defined by (12):

$$
\begin{aligned}
\tau_{x y, \tau}^{(1)}(t) & =B_{3} s_{\tau}^{(1)}+B_{4} \beta_{\tau}^{(1)}+B_{5} \frac{\partial^{2} s_{\tau}^{(1)}}{\partial y^{2}}+B_{6} \frac{\partial^{2} \beta_{\tau}^{(1)}}{\partial y^{2}}-C_{2} \frac{\partial^{2} \psi_{\tau}^{(1)}}{\partial y^{2}}+C_{3} \frac{\partial v_{x, \tau}^{(1)}}{\partial y} \\
& =E \tau_{x y, v}^{(1)}\left(t-\frac{\chi}{\omega}\right) \\
& =E\left[\tau_{1, v} \cos (\omega t-\chi)+\tau_{2, v} \sin (\omega t-\chi)\right] \\
& =E\left[\tau_{1, v}(\cos \omega t \cos \chi+\sin \omega t \sin \chi)+\tau_{2, v}(\sin \omega t \cos \chi-\cos \omega t \sin \chi)\right] \\
& =E^{2}\left[\left(\tau_{1, v}^{2}+\tau_{2, v}^{2}\right) \cos \omega t+(0) \sin \omega t\right] \\
& =\cos \omega t,
\end{aligned}
$$

which is the imposed stress boundary condition.

Similarly, we could have started with imposed stress boundary conditions, and then use the resulting plate velocity components $V_{1, \tau}$ and $V_{2, \tau}$ to compute a phase shift and a rescaling that will lead to the imposed velocity conditions.

Furthermore, the storage and loss moduli are defined by

$$
G^{\prime}(\omega)=\frac{\omega}{2} \frac{\tau_{2} V_{1}-\tau_{1} V_{2}}{V_{1}^{2}+V_{2}^{2}}, \quad G^{\prime \prime}(\omega)=\frac{\omega}{2} \frac{\tau_{1} V_{1}+\tau_{2} V_{2}}{V_{1}^{2}+V_{2}^{2}} .
$$

For imposed velocity boundary conditions, $V_{1, v}=1$ and $V_{2, v}=0$ so that we have $G_{v}^{\prime}(\omega)=$ $\frac{\omega}{2} \tau_{2, v}$. For imposed stress, $V_{\tau}(t)=E V_{v}\left(t-\frac{\chi}{\omega}\right)=E \cos (\omega t-\chi)$, and so $V_{1, \tau}=E \cos \chi$ and $V_{2, \tau}=E \sin \chi$. Thus, $G_{\tau}^{\prime}(\omega)=\frac{\omega}{2} \frac{-V_{2, \tau}}{V_{1, \tau}^{2}+V_{2, \tau}^{2}}=\frac{-\omega \sin \chi}{2 E}=\frac{\omega}{2} \tau_{2, v}$. The proof that $G_{\tau}^{\prime \prime}(\omega)=$ $G_{v}^{\prime \prime}(\omega)=\frac{\omega}{2} \tau_{1, v}$ is similar.

Therefore, even though we may not yet have determined the exact value of the shear stress components, we have shown that the solution for imposed velocity is equal to a phase shift and a rescaling of the solution with imposed stress, and therefore that the dynamic moduli are independent of the choice of boundary conditions. 


\section{References}

de Andrade Lima LRP, Rey AD (2004) Assessing flow alignment of nematic liquid crystals through linear viscoelasticity. Phys Rev E 70:011701

de Andrade Lima LRP, Rey AD (2006) Superposition principles for small amplitude oscillatory shearing of nematic mesophases. Rheol Acta 45: 591-600

Burghardt WR (1991) Oscillatory shear flow of nematic liquid crystals. J Rheol 35: 49-62

Choate EP, Cui Z, Forest MG (2008) Effects of strong anchoring on the dynamic moduli of heterogeneous nematic polymers. Rheol. Acta 47: 223-236

Choate EP, Forest MG (2006) A classical problem revisited: Rheology of nematic polymer monodomains in small amplitude oscillatory shear. Rheol Acta 46: 83-94

Forest MG, Wang Q (2003) Monodomain response of finite-aspect-ratio macromolecules in shear and related linear flows. Rheol Acta 42: 20-46

polymers

Mendil H, Baroni P, Noirez L (2005) Unexpected giant elasticity in side-chain liquid-crystal polymer melts: A new approach for the understanding of shear-induced phase transition. Europhys Lett 76: 983-989

Mendil H, Baroni P, Noirez L (2006) Solid-like rheological response of non-entangled polymers in the molten state. Euro Phys J E 19:77-85

Pujolle-Robic C, Noirez L (2001) Observation of shear-induced nematic-isotropic transition in side-chain liquid crystal polymers. Nature 409: 167-171

Wang Q (2002) A hydrodynamic theory of nematic liquid crystalline polymers of different configurations. J Chem Phys 116: 9120-9136 